\title{
Bradycardia as a rare symptom of cisplatin cardiotoxicity: A case report
}

\author{
JAKUB KUCHARZ ${ }^{1,2}$, ANNA MICHALOWSKA-KACZMARCZYK ${ }^{1}$, ANETA L.ZYGULSKA ${ }^{1}$, \\ JOLANTA WOJTAK $^{3}$, WIESLAW PAWLIK ${ }^{3}$, ROMAN M. HERMAN ${ }^{2}$ and KRZYSZTOF KRZEMIENIECKI ${ }^{1,4}$ \\ ${ }^{1}$ Department of Oncology, University Hospital in Kraków, Kraków 31-531; \\ ${ }^{2}$ Department of Experimental and Clinical Surgery, Jagiellonian University Medical College, Kraków 31-126; \\ ${ }^{3}$ Section of Computed Tomography, Second Department of Internal Medicine, University Hospital in Kraków, Kraków 31-066; \\ ${ }^{4}$ Department of Oncology, Jagiellonian University Medical College, Kraków 31-531, Poland
}

Received December 20, 2014; Accepted December 16, 2015

DOI: $10.3892 / \mathrm{ol} .2016 .4195$

\begin{abstract}
Cisplatin (DDP) is one of the most frequently used chemotherapeutic agents, and has a characteristic toxicity profile. For DDP, complications affecting the cardiovascular system, which are typical for certain other agents, are rare; however, their occurrence may lead to life-threatening conditions. To the best of our knowledge, there are few reported cases of DDP-induced bradycardia in the relevant medical literature. The current report presents the case of a 58-year-old patient diagnosed with metastatic neuroendocrine carcinoma with a primary lesion in the posterior mediastinum, who was treated with DDP and etoposide chemotherapy. Following the initial chemotherapy cycle, the patient experienced severe symptomatic bradycardia (a drop in heart rate to $40 \mathrm{bpm}$ ), with the corrected QT interval prolonged to $424 \mathrm{msec}$. The patient's condition required close monitoring and treatment. Similar symptoms occurred following each of the three cycles of chemotherapy. Imaging studies performed following the third treatment cycle revealed disease progression, and the patient was referred for palliative care. Reports have indicated that damage to the cardiovascular system, including cardiac ischemia, diastolic disturbances, hypertension and microalbuminuria, may be associated with DDP-based therapy. However, the mechanism of DDP-associated cardiac toxicity remains to be elucidated. It may be induced by factors including direct toxicity, ion imbalance, heart infiltration and, in the case of neuroendocrine tumors, the influence of tumor excretions.
\end{abstract}

\section{Introduction}

Cisplatin (DDP) is one of the most frequently utilized chemotherapeutic agents. The Food and Drug Administration first

Correspondence to: Dr Jakub Kucharz, Department of Oncology, University Hospital in Kraków, 10 Sniadeckich, Kraków 31-531, Poland

E-mail: jakub.kucharz@uj.edu.pl

Key words: cisplatin, bradycardia, arrhythmia, cardiotoxicity approved DDP for the treatment of ovarian and testicular cancer in 1978 (1). Since then, therapeutic indications for DDP use (in single-drug and combination therapies) have been significantly extended. DDP is currently used for the treatment of lung, head and neck, and ovarian cancers, as well as germ cell tumors.

DDP has a characteristic toxicity profile that includes nephrotoxicity, neurotoxicity and ototoxicity (2). It is additionally a highly emetogenic agent, particularly in doses $>50 \mathrm{mg} / \mathrm{m}^{2}$ (2). Complications affecting the cardiovascular system, whilst typical for various other agents (including anthracyclines, 5-fluorouracil, taxanes and targeted drugs including trastuzumab, lapatinib or bevacizumab), are rare with DDP treatment (3). Advancements in the understanding of cardio-oncology are important due to the increased frequency of use of combination therapies, the cardiotoxicity of novel therapies (including targeted therapies that interfere with physiological signaling pathways), and the continually increasing rates of long-term survival of patients, for whom delayed treatment-associated complications may occur (4).

As cardiotoxicity is not a typical side-effect of DDP, the present study reports a case of cardiac arrhythmia occurring in a patient with a metastatic neuroendocrine carcinoma of the posterior mediastinum, which was treated with etoposide and DDP (EP regimen) chemotherapy.

\section{Case report}

In September 2012, a 58-year-old Caucasian man was referred from the Pulmonary Hospital Zakopane (Zakopane, Poland) to the Department of Oncology, University Hospital in Kraków (Kraków, Poland), due to metastatic neuroendocrine carcinoma, with a primary lesion in the posterior mediastinum. Histopathological examination performed prior to referral to University Hospital in Kraków revealed a large cell neuroendocrine carcinoma, which was positive for cluster of differentiation 56, chromogranin, synaptophysin and cytokeratin 7, and had a Ki-67 index of $80 \%$.

The histopathological findings and metastatic stage of the disease qualified the patient for palliative chemotherapy with an EP regimen $\left(100 \mathrm{mg} / \mathrm{m}^{2}\right.$ etoposide, days $1-3 ; 25 \mathrm{mg} / \mathrm{m}^{2} \mathrm{DDP}$, 
days 1-3). Computed tomography (CT; Aquilion 64 CT scanner; Toshiba, Tokyo, Japan) imaging of the chest revealed a posterior mediastinum tumor measuring $16.5 \times 13.0 \times 13.5 \mathrm{~cm}$. The tumor infiltrated the aorta and the branch of the right pulmonary artery, with subsequent obstruction of the lower right pulmonary vein, compression of the atria and constriction of the right middle and inferior bronchus. Numerous metastatic lesions were identified in the lungs (Figs. 1 and 2).

Upon admission, the patient complained of heart palpitations and mild resting dyspnea. The patient's Eastern Cooperative Oncology Group performance score was 1 (5), and physical examination revealed tachycardia $(\sim 110 \mathrm{bpm}$; normal range, 60-100 bpm). Laboratory analysis did not reveal any significant deviations from normal values. An electrocardiogram (ECG; FX 2000; Emtel, Zabrze, Poland) revealed sinus tachycardia of $110 \mathrm{bpm}$, a left axis deviation, a left anterior hemiblock (LAHB) and a corrected QT (QTc) interval of $410 \mathrm{msec}$. The decision was made to administer $25 \mathrm{mg}$ of immediate-release metoprolol, twice per day over two days. This reduced the patient's heart rate to $80 \mathrm{bpm}$. The patient was discharged from hospital in a stable condition with beta-blockers. The first cycle of EP was initiated on the following day.

Following the initial $48 \mathrm{~h}$ of chemotherapy, the patient exhibited resting dyspnea, chest discomfort, a heart rate reduction to $40 \mathrm{bpm}$ (normal range, 60-100 bpm) and blood pressure of 130/80 mmHg (normal range, 90/60-140/90 mmHg). ECG revealed sinus bradycardia of $40 \mathrm{bpm}$, left axis deviation, LAHB and a QTc interval of $424 \mathrm{msec}$ (normal range, $<440 \mathrm{msec}$ ), with none of the typical changes for acute coronary syndrome. Laboratory tests were negative for markers of cardiac necrosis; potassium, calcium and magnesium levels were all within normal ranges. The patient was administered intravenous atropine $(0.50 \mathrm{mg}$ every $6 \mathrm{~h})$, which raised the heart rate to $\sim 70 \mathrm{bpm}$, and metoprolol treatment was discontinued. Due to suspected infiltration of the tumor into the electrical conduction system of the heart, an echocardiogram was performed, which revealed that the ejection fraction of the left ventricle was $61 \%$ (normal range, 55-70\%), and the right ventricle systolic pressure was $43 \mathrm{mmHg}$ (normal range, $15-30 \mathrm{mmHg}$ ). There was an insignificant amount of fluid in the pericardium, and a constriction of the inferior vena cava to $6 \mathrm{~mm}$ at its outlet into the atrium, with no clear infiltration into the heart structures.

During the subsequent three days, the patient was administered oral atropine $(0.25 \mathrm{mg}$ every $8 \mathrm{~h}$ ), which led to a gradual improvement of his clinical state and raised his heart rate. On day 4 following the initial occurrence of bradycardia, atropine treatment was ceased and the patient's heart rate was $\sim 110 \mathrm{bpm}$. The patient was seen by a consultant in internal medicine, and the suggestion to re-administer the beta-blocker drug was made (single $25 \mathrm{mg}$ dose of extended-release metoprolol per day). As a consequence of this therapy, the patient's heart rate was reduced to $80-90 \mathrm{bpm}$. The patient was discharged from hospital in a stable and relatively good condition. Subsequently, the patient was admitted for the next course of chemotherapy. Due to the potential chemosensitivity of the tumor and a lack of alternative treatment options, the decision was made to continue chemotherapy despite the previous complications. Episodes of bradycardia ( $40 \mathrm{bpm})$, were again observed during the second and third treatment

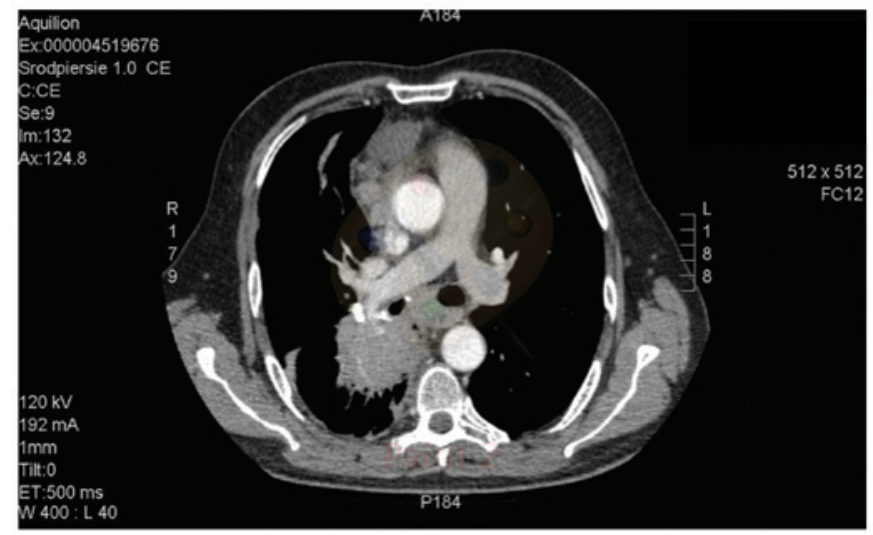

Figure 1. Computed tomography image of the mediastinal mass and lung metastatic lesions.

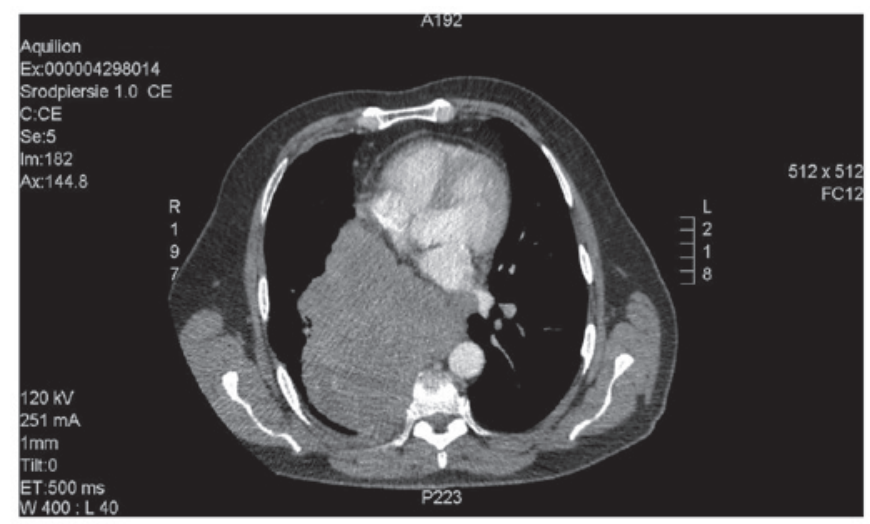

Figure 2. Computed tomography image of the tumor mass.

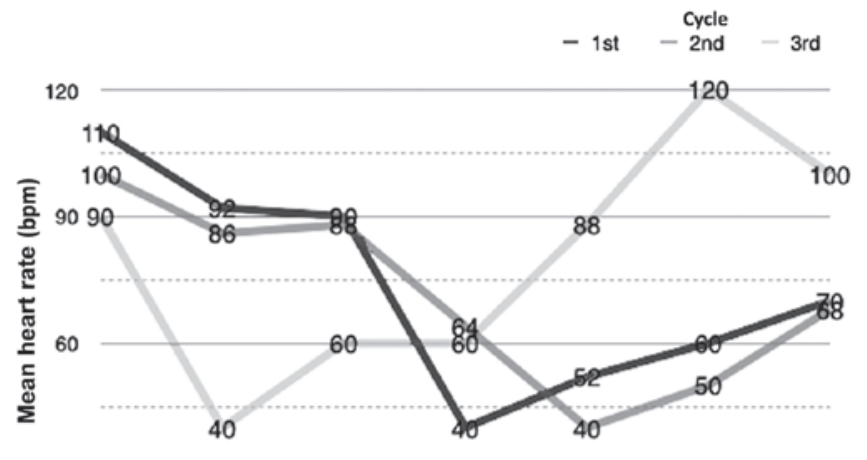

30

40

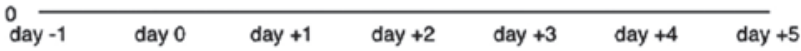

Figure 3. Mean heart rate on the day prior to chemotherapy, days during chemotherapy administration (days 0-2), and days following the termination of chemotherapy.

cycles. These episodes occurred on the third and second days of chemotherapy, respectively.

Identical cardiac treatments to those given during the first cycle were administered. A CT scan of the chest, performed after the third treatment cycle, revealed disease progression according to the Response Evaluation Criteria in Solid 
Tumors 1.1.guidelines (6). The ineffectiveness and the patient's low tolerance of the treatment resulted in termination of the chemotherapy. The patient was referred to a local palliative care center and lost to follow-up.

\section{Discussion}

Cardiotoxicity may be an early or late complication in the systemic treatment of cancer. Early complications are frequently associated with pathophysiological mechanisms, including coronary artery contraction, effects on the autonomic nervous system or electrolyte disturbances. Late complications are primarily associated with the development of cardiomyopathy (7).

Damage to the cardiovascular system, including cardiac ischemia, diastolic disturbances, hypertension and microalbuminuria, has been reported in association with DDP-based therapy (7). These symptoms have been described for patients who underwent testicular cancer therapy with DDP-based regimens (7). Tassinari et al (8) described three cases of patients experiencing sudden atrial fibrillation following DDP infusion. In the available literature, to the best of our knowledge, there have been only two descriptions of DDP-induced bradycardia $(9,10)$. Schlaeffer et al (9) described a patient with nasopharyngeal squamous cell carcinoma, whose pulse dropped each time combination chemotherapy with cisplatin was administered, and the symptoms of the patient became worse; no other possible causes of bradycardia were observed. Altundağ et al (10) presented the case of a patient with relapsed Hodgkin's lymphoma that was treated with cisplatin-based salvage chemotherapy. Asymptomatic bradycardia was observed in the patient on days 3-5 following the first cycle of treatment and on days 2-5 following subsequent treatment cycles. The patient did not require any treatment for bradycardia, and a dose reduction or chemotherapy discontinuation was not required. The mechanism of DDP-associated cardiac arrhythmia remains to be elucidated. It may be induced by various factors, including intracellular and extracellular fluctuations of $\mathrm{K}^{+}$and $\mathrm{Mg}^{2+}$ concentrations caused by DDP (11), or by the accumulation of DDP in the electrical conduction system of the heart (for example, in the cells of the sinoatrial node), which may lead to electrical transmission disturbances (7).

Additional pathological mechanisms may include infiltration involving the cardiac muscle or the electrical conduction system of the heart, infiltration affecting the vagus nerve and, in neuroendocrine tumors, the influence of tumor excretions, which may affect the cardiovascular system directly or indirectly and should be taken into account in patients with malignancies located within the mediastinum (12). The toxic influence of DDP on the cardiac muscle appears to be the most likely cause of arrhythmia in the present case. This is supported by the disturbances recorded by the ECG, and by the temporal association observed between the administration of DDP and the onset of bradycardia (Fig. 3). In summary, the present case suggests that medical oncologists should be aware of the cardiotoxicity of DDP, as it may occur in patients with no pre-existing cardiac disease. Particular attention should be paid to patients with a history of arrhythmias (in particular bradycardia) and those being administered beta-adrenolytics or non-dihydropyridine calcium channel blockers, as such patients are prone to bradycardia.

\section{References}

1. Prestayko AW, D'Aoust JC, Issell BF and Crooke ST: Cisplatin (cis-diamminedichloroplatinum II). Cancer Treat Rev 6: 17-39, 1979.

2. Loehrer PJ and Einhorn LH: Drugs five years later. Cisplatin. Ann Intern Med 100: 704-713, 1984

3. Bovelli D, Plataniotis G and Roila F; ESMO Guidelines Working Group: Cardiotoxicity of chemotherapeutic agents and radiotherapy-related heart disease: ESMO Clinical Practice Guidelines. Ann Oncol 21 (Suppl 5): v277-v282, 2010.

4. Yeh ET, Tong AT, Lenihan DJ, Yusuf SW, Swafford J, Champion C, Durand JB, Gibbs H, Zafarmand AA and Ewer MS: Cardiovascular complications of cancer therapy: Diagnosis, pathogenesis, and management. Circulation 109: 3122-3131, 2004

5. Oken MM, Creech RH, Tormey DC, Horton J, Davis TE, McFadden ET and Carbone PP: Toxicity and response criteria of the Eastern Cooperative Oncology Group. Am J Clin Oncol 5: 649-655, 1982.

6. Eisenhauer EA, Therasse P, Bogaerts J, Schwartz LH, Sargent D, Ford R, Dancey J, Arbuck S, Gwyther S, Mooney M, et al: New response evaluation criteria in solid tumours: Revised RECIST guideline (version 1.1). Eur J Cancer 45: 228-247, 2009.

7. Meinardi MT, Gietema JA, van der Graaf WT, van Veldhuisen DJ, Runne MA, Sluiter WJ, de Vries EG, Willemse PB, Mulder NH, van den Berg MP, et al: Cardiovascular morbidity in long-term survivors of metastatic testicular cancer. J Clin Oncol 18: 1725-1732, 2000

8. Tassinari D, Sartori S, Drudi G, Panzini I, Gianni L, Pasquini E, Abbisciano V, Ravaioli A and Iorio D: Cardiac arrhythmias after cisplatin infusion: Three case reports and a review of the literature. Ann Oncol 8: 1263-1267, 1997.

9. Schlaeffer F, Tovi F and Leiberman A: Cisplatin-induced bradycardia. Drug Intell Clin Pharm 17: 899-901, 1983.

10. Altundağ O, Celik I and Kars A: Recurrent asymptomatic bradycardia episodes after cisplatin infusion. Ann Pharmacother 35: 641-642, 2001.

11. Blachley JD and Hill JB: Renal and electrolyte disturbances associated with cisplatin. Ann Intern Med 95: 628-632, 1981.

12. Maier HC and Sommers SC. Neuroendocrine carcinoma of lung associated with bradycardia and episodic cardiac asystole. Ann Thorac Surg 41: 560-562, 1986. 